\title{
LA OPINIÓN DE LOS PROFESIONALES DE LOS CENTROS PENITENCIARIOS DE ANDALUCÍA SOBRE LOS MÓDULOS DE RESPETO
}

\author{
THE OPINION OF PRISON-SERVICE PROFESSIONALS IN ANDALUCIA \\ REGARDING “RESPECT DEPARTMENTS”
}

\section{A OPINIÃO DOS PROFISSIONAIS DOS ESTABELECIMENTOS PRISIONAIS DA ANDALUZIA SOBRE OS MÓDULOS DE RESPEITO}

\author{
Francisco FERNÁNDEZ MILLÁN* \& Purificación PÉREZ-GARCÍA**
}

* Centro Penitenciario de Albolote (Granada, España), **Universidad de Granada

\begin{tabular}{|c|c|}
\hline & $\begin{array}{r}\text { Fecha de recepción del artículo: } 25.1 \mathrm{~V} .2017 \\
\text { Fecha de revisión del artículo: 6.VI.2017 } \\
\text { Fecha de aceptación final: 10.VII.2017 }\end{array}$ \\
\hline $\begin{array}{l}\text { PALABRAS CLAVE: } \\
\text { funcionario de } \\
\text { prisiones } \\
\text { equipo técnico } \\
\text { mando directivo } \\
\text { prisión } \\
\text { tratamiento } \\
\text { penitenciario }\end{array}$ & $\begin{array}{l}\text { RESUMEN: El grupo de profesionales que trabajan en los Módulos de Respeto está poco } \\
\text { estudiado. Son un sistema de organización interna de la prisión. Nuestro objetivo era conocer } \\
\text { su posicionamiento sobre estos módulos; y de forma específica, averiguar si había alguna } \\
\text { relación entre el concepto de estos y el grupo profesional al que se pertenecía: funciona- } \\
\text { rio, equipo técnico y mando directivo. Nos apoyamos en el método descriptivo y aplicamos } \\
\text { un cuestionario de elaboración propia "Cuestionario para Profesionales sobre los Módulos } \\
\text { de Respeto", al que sometimos al alpha de Cronbach para la fiabilidad (.766) y al análisis de } \\
\text { componentes principales para su validez de contenido que explicó el 61.102\% de la varianza. } \\
\text { Participó una muestra de } 315 \text { sujetos de las ocho provincias andaluzas de los tres sectores. } \\
\text { Se aplicó el análisis de contingencias y los resultados mostraron que los Módulos de Respeto, } \\
\text { como herramienta formativa y lo que se entendía por este concepto, se relacionaban de for- } \\
\text { ma distinta en función del sector profesional. }\end{array}$ \\
\hline $\begin{array}{l}\text { KEY WORDS: } \\
\text { prison officer } \\
\text { technical team } \\
\text { managerial team } \\
\text { prison } \\
\text { penitentiary } \\
\quad \text { treatment }\end{array}$ & $\begin{array}{l}\text { ABSTRACT: Relatively few studies have focused on the group of professionals who work in } \\
\text { the Respect Departments, which are internal units within the prison. Our aim was to under- } \\
\text { stand the opinion of prison professionals in relation to these modules, and, in particular, to } \\
\text { identify if there was any relationship between these opinions and the professional group to } \\
\text { which they belonged, including prison officers, professionals such as psychologists, psychi- } \\
\text { atrists, educators, and other members of the technical and management teams. This study } \\
\text { used the descriptive method, developing our own questionnaire titled "Questionnaire for } \\
\text { Professionals on the Respect Departments", which was tested for reliability using Cronbach's }\end{array}$ \\
\hline
\end{tabular}

CONTACTAR CON LOS AUTORES: Purificación PÉREZ-GARCíA. Campus Universitario de Cartuja. Facultad de Ciencias de la Educación s/n 18071 Granada. mpperez@ugr.es 


\begin{tabular}{l|l}
\hline & $\begin{array}{l}\text { alpha (.766) whilst validity of its main components was analyzed, which explained 61.102\% of } \\
\text { the variance. A sample of } 315 \text { subjects from the eight Andalusian provinces of the three sec- } \\
\text { tors participated in the study. Contingency analysis was applied and the results revealed that } \\
\text { the Respect Departments were viewed differently both as an educative tool and in general, } \\
\text { depending on the group to which the prison staff belonged. }\end{array}$ \\
\hline $\begin{array}{l}\text { PALAVRAS-CHAVE: } \\
\text { puncionário de } \\
\text { equipa técnica } \\
\text { coordenação diretiva } \\
\text { risão } \\
\text { tratamento prisional }\end{array}$ & $\begin{array}{l}\text { RESUMO: O grupo de profissionais que trabalham nos Módulos de Respeito encontra-se } \\
\text { pouco estudado. São um sistema de organização interna da prisão.O nosso objetivo era co- } \\
\text { nhecer o seu posicionamento sobre estes módulos; e de forma específica, averiguar seexistia } \\
\text { alguma relação entre o conceito destes eo grupo profissional ao qual pertenciam: funcionário, } \\
\text { equipa técnico ecoordenaçãodiretiva. Apoiámo-nosno método descritivo e aplicámos um- } \\
\text { questionário de elaboração própria “Questionário para Profissionais sobre os Módulos de } \\
\text { Respeito", ao qual submetemos o alpha de Cronbach para a fiabilidade (.766) ea análise das } \\
\text { componentes principais para a sua validade de conteúdo, que explicou os 61.102\% da varia- } \\
\text { ção. A participação contou com uma amostra de } 315 \text { sujeitos das oito províncias andaluzas, } \\
\text { dos três sectores. Foi aplicada a análise das contingências e os resultados demostraram que } \\
\text { os Módulos de Respeito, como ferramenta formativa eo que se entendia por este conceito, } \\
\text { se relacionavam de forma distinta na função do sector profissional. }\end{array}$ \\
\hline
\end{tabular}

\section{Introducción}

El mundo penitenciario es un ámbito muy estudiado en nuestro país desde diversas perspectivas (psicológica, sociológica, criminológica y educativa) de las que Martín, Vila y De Oña (2013) han hecho una magnífica revisión, dejando al descubierto cuál es el estado de la investigación educativa en este medio, en nuestro país.

Los estudios se centran principalmente sobre la población penitenciaria (efecto de la prisionalización, droga, género, mayores, problemas mentales,...) (Añaños, 2017; García-Legaz \& Crespo, 2014; Martín, 2008), muy pocos sobre los Módulos de Respeto (en adelante $\mathrm{MdR}$ ) y menos aún sobre los profesionales que trabajan en estos módulos, a saber, los funcionarios de vigilancia y los equipos técnicos.

La investigación que presentamos es el primer estudio en la comunidad autónoma andaluza, que se preocupa de explorar la opinión sobre los MdR que poseen los profesionales de los centros penitenciarios, sabiendo que se necesita la máxima implicación para su éxito.

En el ámbito nacional, escasea la investigación sobre los profesionales que desarrollan su labor en estos módulos. Hemos encontrado sobre el ámbito penitenciario el número 22 de 2016 de la "Revista de Educación Social. (RES)"; el monográfico de 2013 de la "Revista de Educación"; el número 22 de 2013 de la Revista de "Pedagogía Social. Revista Interuniversitaria" y el número 59 de 2014 de la "Revista Galega de Educación". Las únicas referencias específicas sobre profesionales en cárceles en general, pero no en los $\mathrm{MdR}$, son los estudios de Antón (1998) y Valderrama (2010).

En el ámbito internacional no se encuentran referencias a los MdR, porque es un sistema específicamente español (Belinchón, 2009), surgido en 2001, que ahora se está exportando a algunos países europeos como Francia e Inglaterra. Existen experiencias, ya desde los años setenta, que comparten la finalidad socializadora de los $\mathrm{MdR}$, aunque no siguen ni su estructura ni su organización. En la última década, algunas de estas prácticas se caracterizan por conformar programas de mentorización (Cook, McClure, Koutsenok \& Lord, 2008; Marlow, Grajeda, Lee, Young, Williams \& Hill, 2015), programas de terapia social (Suhling \& Guéridon, 2016), reintegración social a través del trabajo (Novo-Corti \& Barreiro-Gen, 2015) o la apuesta por una aplicación sociológica (Guy, 2011).

\section{Módulos de respeto: profesionales y sentido}

La jurisprudencia aboga por la reinserción, pero ¿y la reeducación? En la actualidad, la Ley fundamental que da cuerpo a toda la regulación en materia penitenciaria es la Ley Orgánica 1/1979, de 26 de septiembre, General Penitenciaria (en adelante LOGP)(BOE no 239, de 5 de octubre). Establece como finalidad fundamental de las penas privativas de libertad la reeducación y reinserción social de los condenados.

Así también se recoge en el art.25.2 de la Constitución española, que establece que las medidas de seguridad estarán orientadas hacia la reeducación y reinserción social (Añaños, Fernández-Sánchez \& Llopis, 2013; Cervelló, 2016). De ambas expresiones,

la primera recibe mayor rechazo por implicar interiorización de valores, lo que hace que tenga más aceptación la segunda como reincorporación social alejada del delito, entendiéndola no tanto como actuaciones directas sobre el sujeto, sino como creación de las condiciones sociales necesarias para producir un menor índice de delincuencia. (Cervelló, 2016, p. 41) 
Las respuestas que se adopten, en cualquiera de los casos, serán actividades "metodológicamente plurales y abiertas a un quehacer pedagógico de amplias miras" (Caride \& Gradaílle, 2013, p. 36). Tales respuestas se concretan, de forma operativa, en el tratamiento penitenciario. Pueden ser de tipo médico, psiquiátrico, psicológico, pedagógico, laboral o social. Aunque no está sistematizado por la LOGP, se alude a la psicoterapia de grupo, asesoramiento pedagógico y terapia de comportamiento, actividades como el trabajo, el deporte, la educación, la cultura o el ocio.

Los MdR, entendemos, son el germen que ha propiciado el planteamiento de programas que reeducan y resocializan, superando enfoques terapéuticos y clínicos (Añaños \& Yagüe, 2013). El nuevo rumbo ha de orientarse más hacia un sentido educativo y moral de la actuación (Gil, 2010, 2016). Esto implica compromiso con los internos por parte de todos los profesionales. Es decir, han de comprenderse, ambos, sin enjuiciarse, con el único objetivo de mejorar como personas y querer vivir conforme a la ley. Por tanto, los MdR son una metodología que ofrece futuro dentro del centro penitenciario.

Se definen como "un programa de intervención con instrumentos, dinámicas, estructuras y pautas de actuación y evaluación definidas y sistematizadas" (Belinchón \& García, 2014, p. 162). Para Valderrama (2016, p. 31) son estructuras organizativas internas que "partiendo de la voluntariedad y del compromiso en la participación, tanto en actividades como en el funcionamiento del propio módulo, tienen como fin generar espacios flexibles, favorecedores de un adecuado clima de convivencia y posibilitadores de programas de intervención".

Las experiencias resocializadoras y reeducadoras en el ámbito penitenciario español son escasas, pero variadas. Del Pozo y Añaños (2013) apuestan por programas de convivencia (habilidades sociales y personales, autonomía y liderazgo), de interculturalidad y de valores. Valderrama (2016) lidera un proyecto basado en un enfoque dialógico-comunicativo, generando mecanismos de gestión compartida. Lorenzo, Aroca y Alba (2013) se detienen en el Programa de Pensamiento Prosocial que, en el contexto español, se ha aplicado con población variada. La experiencia del Programa Good Lives (Gil, 2013) es otra vía posible de reeducación cuyo fundamento se arraiga en un marco legislativo, ético y antropológico de los derechos humanos. Es decir, se trata de pensar en la potencialidad de los derechos humanos desde una perspectiva jurídica, que favorezca las reformas legislativas penitenciarias y la gestión de las prisiones; $y$ de las posibilidades educativas de los derechos humanos y del derecho a la educación en los contextos penitenciarios.

Para la resocialización y reeducación hemos de contar con la administración penitenciaria. Esta se organiza en torno a órganos unipersonales y órganos colegiados con funciones perfectamente delimitadas. Los primeros están compuestos por el director, subdirector, administrador, jefe de servicio; y en los segundos, el consejo de dirección, la junta de tratamiento, el equipo técnico, la comisión disciplinaria y la junta económico-administrativa. Sin estar clasificado dentro de ninguno de estos órganos, desarrolla su labor dentro del centro penitenciario, el cuerpo de funcionarios de vigilancia.

En nuestro estudio, nos interesa comprender la labor de los distintos profesionales para interpretar el ámbito penitenciario y el sentido con el que es analizado. El director es la máxima figura. Sus funciones consisten en dirigir, coordinar $y$ supervisar las directrices relativas a la vida de la prisión, como tratamiento, régimen, sanidad, personal, de gestión económico-administrativa, de representación y de orden disciplinario (Cervelló, 2016).

El equipo técnico se encarga de atender la vida del recluso en la cárcel. Lo que se materializa con la ejecución de los programas de tratamiento o modelos individuales de intervención. Podrán estar compuestos por jurista, psicólogo, pedagogo, sociólogo, médico, ayudante técnico sanitario/ diplomado universitario en enfermería, maestro o encargado de taller, educador, trabajador social, monitor sociocultural o deportivo y encargado de departamento. Aunque, como expone Valderrama (2016, p. 34), "los perfiles profesionales que actualmente configuran el cuerpo de técnicos penitenciarios son básicamente el de juristas y psicólogos/as, existiendo una ausencia generalizada de pedagogos/as". Se reivindica la figura del educador social que, en su actuación, supera enfoques terapéuticos, legalistas y criminológicos, "centrándose en diseñar actividades de ocupación exigentes favorecedoras del cambio personal y social, tanto en actitudes como en valores" (Gil, 2016, p. 61).

La Dirección General de Instituciones Penitenciarias (DGIP, 2007) indica que, en los MdR, las funciones del equipo técnico se concretan en: a) reuniones semanales en sesiones informales; b) evaluación de los internos y la marcha del programa; y c) establecimiento de normas y organización; recomendando coordinación entre el equipo, los funcionarios y los internos. Por otro lado, estos equipos también tienen la misión de fomentar, motivar y ubicar al preso en el interior del módulo, en base al perfil individualizado de cada interno (Cendón, Belinchón \& García, 2011). 
Los funcionarios de interior o vigilancia se aseguran de mantener la seguridad y el orden dentro de la prisión. La DGIP (2007) entiende que deben ser voluntarios y formados en la dinámica de funcionamiento del MdR. Sus funciones son: a) responsable de la realización de las actividades por parte de los internos; b) conocedor de su programación; c) evaluación diaria de cada interno; y d) participación en las reuniones semanales del Equipo Técnico.

Pero la propia DGIP manifiesta cierto temor, ya que, a pesar de estar estipuladas sus funciones, de haber menor conflictividad, de tener un rol más positivo y cambiar el tipo de relación interpersonal, - pues aquí ya no es el enemigo del preso que "le limita la poca libertad de la que dispone" (Galán, 2015, p. 366), sino que al contrario, supone un acercamiento-, y de dignificar su función, es posible encontrar resistencia o al menos no todo el apoyo incondicional hacia los MdR. El origen proviene de la confusión del rol que ellos han de jugar y por ideas equivocadas sobre el concepto de los MdR.

Por parte de Instituciones Penitenciarias, por tanto, es necesario transmitir a aquellos profesionales que van a desarrollar su trabajo en MdR, la filosofía del sistema, sus ventajas, tanto desde el punto de vista de la finalidad de la Institución Penitenciaria, como para su satisfacción profesional, así como el importante papel que juegan como profesionales en el funcionamiento del mismo (Cendón, Belinchón \& García, 2011).

Después de todo lo expuesto, los profesionales implicados más directamente en los $\mathrm{MdR}$, a saber, los educadores y los funcionarios de vigilancia, centrarán su atención en dos asuntos: el primero, en la situación como problema social de un colectivo y no en el sujeto como problema en sí (Valderrama, 2013), internos como personas con potencialidad para modificar valores y actitudes (Gil, 2016, 2010); y el segundo, en la formación (Añaños \& Yagüe, 2013). Defendemos que ambos han de preocuparse por conocer instrumentos $y$ técnicas que desarrollen su trabajo de forma adecuada, pero sobre todo, "comprometida".

También reconocemos que, la profesionalización en los procesos de diagnóstico, ejecución y evaluación que se han de llevar a cabo en la reeducación y resocialización, no están claramente asignados a los distintos profesionales (Valderrama, 2016). La intervención especializada permite la coherencia y efectividad de esta y es la apuesta a seguir (Del Pozo, 2013).

En cualquier caso, si por algo han de destacar los mandos, los equipos técnicos y los funcionarios de vigilancia es por "la responsabilidad de comprender para intervenir mejor" (Scarfó,
Breglia \& López, 2016, p. 85). Independientemente del conocimiento profesional que posean, al final, el sentido de la actuación "dependerá de su ética, de sus valores y de sus principios" (Expósito \& Llopis, 2016, p. 81) en el quehacer diario dentro de los MdR.

En este sentido, como objetivo de investigación, pretendemos explorar la opinión de los distintos profesionales que trabajan en el ámbito penitenciario (funcionarios de vigilancia, equipo técnico y mando directivo) sobre los MdR.

\section{Metodología}

Los criterios de inclusión para participar en el estudio eran pertenecer al sector de los funcionarios (funcionarios de interior o vigilancia), a los equipos técnicos (juristas, psicólogos, pedagogos, educadores y trabajadores sociales) y a los mandos directivos (directores, subdirectores y jefes de servicio). Según los datos de la Relación de Puesto de Trabajo de los centros penitenciarios de Andalucía, esta población era de 2327 sujetos.

La muestra, que fue representativa con un nivel de confianza del 95\%, estuvo conformada por 315 profesionales de los centros penitenciarios andaluces. Fue calculada aplicando el muestreo aleatorio simple, considerando la población finita, de Tagliacarne (1968).

De Almería participaron el 9,5\% del total de la muestra, de Cádiz el 11,1\%, de Córdoba el 8,3\%, de Granada el 11,7\%, de Huelva el 18,1\%, del Jaén el 11,4\%, de Málaga el 19,7\% y de Sevilla el 10,2\%. El $76,2 \%$ eran hombres y un $23,8 \%$ mujeres. Por sectores, el 66,6\% eran funcionarios de vigilancia, el $31,2 \%$ equipo técnico y el $2,2 \%$ mando directivo. En cuanto a la experiencia profesional, el 35,5\% contaba con más de 25 años de servicio, el 27,1\% entre 15 y 25 años y únicamente el 3,2\% presentaba menos de 5 años de antigüedad. Centrándonos en los años que llevaban desarrollando su labor profesional en los MdR, el 52,4\% acumulaba de 1 a 3 años, el 25,4\% entre 3 y 5 años y más de 5 años el $13,3 \%$. El 54,9\% prefería los MdR frente al 31,7\% los módulos ordinarios.

En nuestra investigación recurrimos al método descriptivo (Cohen \& Manion, 1990) y para la recogida de datos, siendo coherentes con el método, un cuestionario (McMillan \& Schumacher, 2005) de diseño propio que titulamos "Cuestionario para Profesionales sobre los Módulos de Respeto". Se estructuraba en tres partes. La primera referida a los datos de identificación: sexo, grupo profesional, sección donde desarrollaba la actividad laboral, experiencia como profesional penitenciario y en MdR, así como dónde preferiría desarrollar su quehacer diario. La segunda 
comprendía el grueso del cuestionario y se dividía en cuatro bloques a los cuales se les aplicó una escala Likert que respondía a las categorías nada 1, poco 2, suficiente 3, bastante 4 y mucho 5: a) posicionamiento general ante los MdR (ítems 1, 2, 8); b) herramienta formativa (ítems 3 al 7); c) utilidad de los MdR (ítems 9 al 12); y d) viabilidad (ítems 13 al 16). La tercera parte se definía con un grupo de seis preguntas abiertas: ¿Qué es lo mejor de los $\mathrm{MdR}$ en relación al resto de departamentos del Centro? ¿Qué es lo peor de los MdR en relación al resto de departamentos del Centro? ¿Qué modificarías de los actuales MdR? ¿Qué eliminarías de los actuales MdR?, y las dos últimas: añade cualquier propuesta de mejora en relación a los MdR que creas importante y necesaria; y añade cualquier propuesta de mejora en relación a la Institución Penitenciaria que creas importante y necesaria.

El cuestionario, de elaboración propia, fue construido tras una revisión de la literatura, académica y legislativa, sobre los MdR. El procedimiento seguido para la recogida de la información fue el cuestionario entregado en mano a los profesionales, ya que contábamos con el permiso del Ministerio del Interior. Fue la Subdirección General de Relaciones Institucionales y Coordinación Territorial con su circular dirigida a los directores de los centros penitenciarios en Andalucía, la que nos abrió paso. Siguiendo los criterios éticos, también se les entregó el consentimiento informado.

Para determinar la validez del cuestionario, recurrimos a la validación estadística mediante el análisis de componentes principales, que clarificó la validez de contenido. A través de este análisis, averiguamos cómo interrelacionaban los ítems (Rodríguez, Olmos \& Martínez, 2012) considerando a partir de .04 el corte (Cea, 2001) para ubicar los ítems en un factor. El valor KMO de adecuación muestral fue de .830 y la esfericidad de Barlett fue significativa con una $p=.000$, teniendo la muestra una adecuada distribución. Obtuvimos 4 factores que explicaban el 61,102 \% de la varianza lo cual nos indicaba el grado de homogeneidad de los datos (Quispe, 2014) en cada uno de los factores, que se ajustaban prácticamente a la estructura del cuestionario presentada. Es decir, el factor "posicionamiento general" mantenía dos de las tres variables $(2,8)$; "herramienta formativa" se reproducía íntegramente con los ítems 3 al 7; el factor "utilidad" conformaba todos los ítems y añadía dos más (1, 15); y el factor "viabilidad" recogía tres de los cuatro ítems.

Para calcular la fiabilidad, siguiendo a Bisquerra (1987), aplicamos el $\alpha$ de Cronbach. Obtuvimos un coeficiente de .766 , indicando una fiabilidad moderada (McMillan \& Schumacher, 2005) y un aceptable grado de consistencia interna, por encima de .600, en palabras de Thorndike (1997).

\section{Resultados}

Abordamos nuestro objetivo, explorando las posibles asociaciones significativas, a través del análisis de contingencia, manejando el paquete estadístico SPSS 20 (Statistical Package for Social Sciences). Para los datos de las preguntas abiertas, practicamos el análisis de contenido (Kelchtermans, 1993), cuya categorización arrojó seis categorías: lo mejor (aspectos positivos de los MdR en relación a otros módulos), lo peor (aspectos negativos de los MdR comparados con otros), a modificar (puntos que habría que cambiar dentro de los MdR), a eliminar (aspectos para suprimir), a mejorar (aspectos que se podrían dejar, pero mejorándolos) y evolución (sentido hacia el que reconducir la filosofía de los MdR).

Para los datos estadísticos (Tabla 1), descubrimos relaciones significativas del sector al que se pertenecía con el posicionamiento ante el concepto de $M d R$ que tenían y con la consideración de los MdR como herramienta formativa. Pero no apareció asociación ninguna sobre la utilidad de los módulos o su viabilidad.

En cuanto al concepto de MdR, tanto los funcionarios de vigilancia como los equipos técnicos valoraron muy positivamente la inclusión y evolución de los MdR en la institución penitenciaria; en esta misma línea, los mandos directivos (57,1\%) valoraron bastante la inclusión y evolución. El mismo patrón encontramos sobre el perjuicio de la creación de MdR sobre el resto de Módulos, donde funcionarios (58\%) y equipo técnico $(45,3 \%)$ opinaron que no perjudicaba nada. En cambio, en mandos directivos había desencuentro, pues un $28,6 \%$ no creía que perjudicara nada y un $28,6 \%$ poco.

Profundizando en los MdR como herramienta formativa averiguamos que los funcionarios creían suficientes $(30,5 \%)$ las actividades de educación o formación académica reglada que se celebraban en el interior de los MdR. Por el contrario, para los equipos técnicos eran muchas (34,7\%). En cambio, no había criterio unánime entre los mandos directivos, pues sus opiniones se repartían, con el mismo porcentaje, en pocas y bastantes.

Los funcionarios de vigilancia (un 40\%) pensaban que en el interior de los MdR no se desarrollaban talleres de trabajo productivo, regulados por el RD 782/2001, por el que los internos estaban asegurados a la Seguridad Social y con nómina mensual. En este sentido, los equipos técnicos $(28,5 \%)$ y los mandos directivos $(42 \%)$ sí 
reconocían que existían, pero mantenían que había pocos.

Las actividades deportivas que se programaban en los MdR, para los tres sectores, eran suficientes, en funcionarios ( $32,9 \%)$, en equipos técni$\cos (38,9 \%)$ y en mandos directivos $(42,8 \%)$.
Que en el interior de los MdR se desarrollaran cursos formativos o de inserción laboral era una afirmación que compartían los funcionarios $(24,7 \%)$ y los equipos técnicos $(28,5 \%)$ para los cuales eran suficientes, frente a los mandos directivos que destacaron que existían pocos $(42,9 \%)$.

\begin{tabular}{|c|c|c|c|c|c|c|c|c|c|}
\hline & & abla 1. & ociaci & s seg & I sectc & e pert & ncia & & \\
\hline 1. ¿Valor & sitivamente la & ión y ev & Eión de & AdR en & stitució & nitencia & & & \\
\hline & & & & & $\%$ & & & $\chi^{2}$ & $p$ \\
\hline & & 1 & 2 & 3 & 4 & 5 & Total & 29,753 & .040 \\
\hline & Funcionario & 9 & 5,3 & 19 & 32,9 & 33,8 & 100 & & \\
\hline Sector & Eq. Técnico & 5,3 & 8,4 & 15,6 & 36,9 & 33,8 & 100 & & \\
\hline & Directivo & 0 & 0 & 0 & 57,1 & 42,9 & 100 & & \\
\hline 3. En el & rior de los Mo & esarroll & ctividad & le educ & o o form & in acade & a reglada & & \\
\hline & & & & & $\%$ & & & $\chi^{2}$ & $p$ \\
\hline & & 1 & 2 & 3 & 4 & 5 & Total & 36,603 & .006 \\
\hline & Funcionario & 14,8 & 9 & 30,5 & 26,7 & 19 & 100 & & \\
\hline Sector & Eq. Técnico & 6,3 & 7,4 & 20 & 31,6 & 34,7 & 100 & & \\
\hline & Directivo & 14,3 & 28,6 & 14,3 & 28,6 & 14,2 & 100 & & \\
\hline $\begin{array}{l}\text { 4. En el } \\
\text { Social y }\end{array}$ & $\begin{array}{l}\text { rior de los } \mathrm{Mc} \\
\text { nómina mens }\end{array}$ & $\begin{array}{l}\text { esarroll } \\
\text { ra los in }\end{array}$ & $\begin{array}{l}\text { alleres c } \\
\text { os) }\end{array}$ & bajo $p$ & ictivo re & do por & $782 / 200$ & egurados & uridad \\
\hline & & & & & $\%$ & & & $\chi^{2}$ & $p$ \\
\hline & & 1 & 2 & 3 & 4 & 5 & Total & 43,689 & .000 \\
\hline & Funcionario & 40 & 17,5 & 14,8 & 17,1 & 10,6 & 100 & & \\
\hline Sector & Eq. Técnico & 17,9 & 28,5 & 17,9 & 16,8 & 18,9 & 100 & & \\
\hline & Directivo & 28,6 & 42 & 0 & 28,6 & 0 & 100 & & \\
\hline 6. En el & rior de los $\mathrm{Mc}$ & esarroll & ctividad & leporti & & & & & \\
\hline & & & & & $\%$ & & & $\chi^{2}$ & $p$ \\
\hline & & 1 & 2 & 3 & 4 & 5 & Total & 42,564 & .001 \\
\hline & Funcionario & 2,9 & 13,2 & 31 & 32,9 & 20 & 100 & & \\
\hline Sector & Eq. Técnico & 2,1 & 9,5 & 15,8 & 38,9 & 33,7 & 100 & & \\
\hline & Directivo & 0 & 28,6 & 14,3 & 42,8 & 14,3 & 100 & & \\
\hline
\end{tabular}




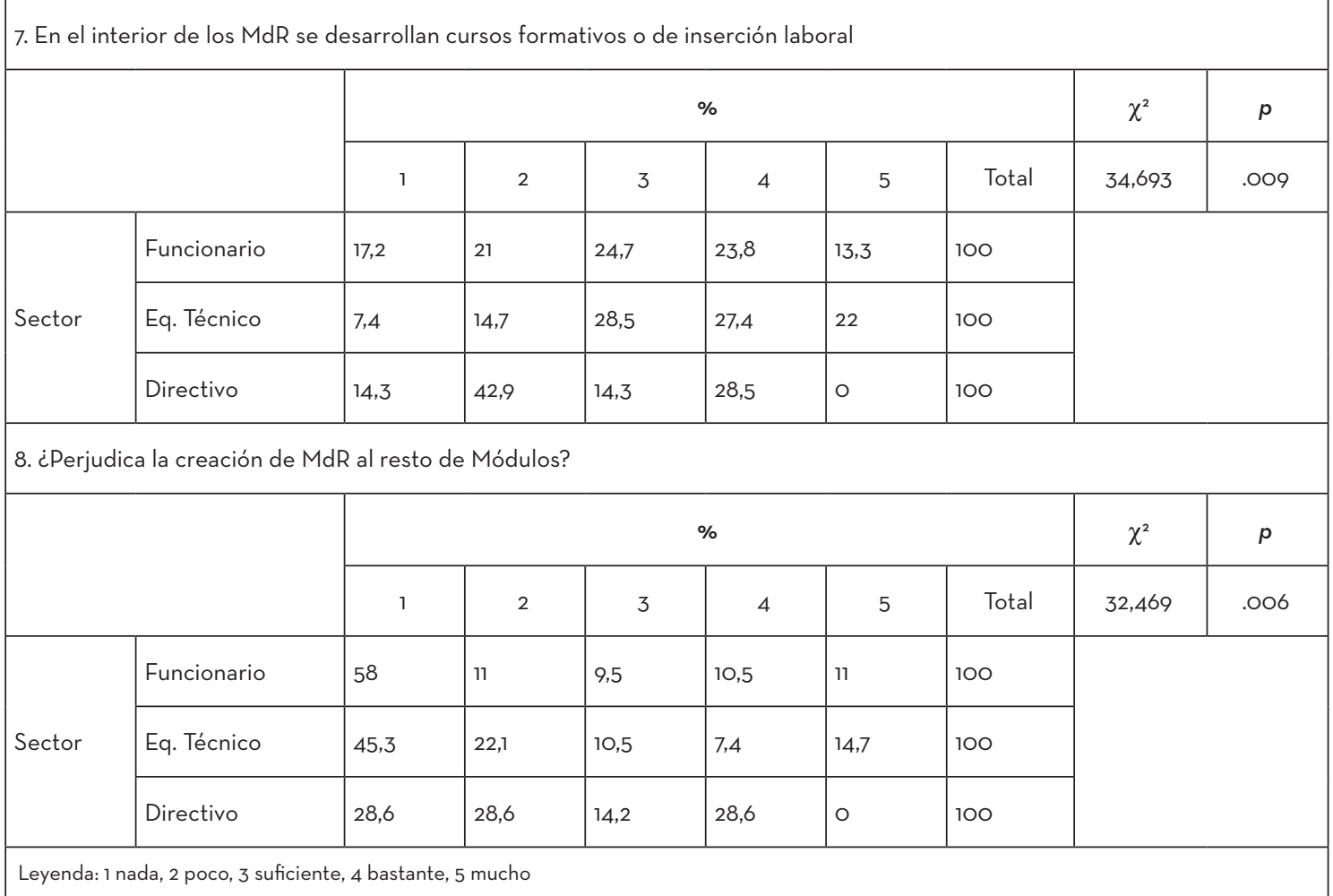

La información obtenida, a través de las preguntas abiertas, la presentamos en función de las 6 categorías extraídas.

En relación a la primera categoría, los tres sectores destacaron como lo mejor de los MdR: la limpieza, inexistencia de conflictos, se acataba la autoridad por parte de los internos, se mejoraba el comportamiento de estos, el clima era silencioso y tranquilo, se proporcionaba más ocupaciones y actividades, se asemejaba más a la vida en libertad, reinaba el orden y la disciplina, predominaba el respeto y la educación, el módulo estaba libre de droga y aumentaba la participación, autonomía y salubridad.

Sobre la segunda, lo peor de los MdR, en opinión de los tres grupos de profesionales era: la burocracia, se relajaba la seguridad, se fomentaba la delación, poco mando de los funcionarios y reducción de sus competencias frente a la mayor autoridad de los equipos técnicos, no se incluían todos los perfiles de presos, no se cumplía el programa tal y como estaba pensado, parecía un "lavado de cara de los centros penitenciarios", los presos se creían con más derechos y menos obligaciones, incluso un interno podía sancionar a otro compañero, el sistema de selección de los internos, se usaba los MdR como "escombrera" para separar presos incompatibles en otros módulos. Era un "paripé y un teatro" de cara a la sociedad, las normas no se adaptaban a la realidad social, la hipocresía y la artificialidad eran evidentes pues se simulaban las conductas.
En la tercera, los puntos a modificar eran: la selección de los presos y su clasificación, así como el acceso y la salida del módulo. También valoraban las relaciones de poder entre el funcionario, la junta de tratamiento y los consejos de dirección. Los funcionarios querían más peso y los equipos técnicos más poder frente al consejo de dirección. Se solicitaba aumentar el número de $M d R$, más recursos, más personal y más actividades intelectuales y menos deportivas, se cuestionaba la sobre-autoridad de los internos y la organización interna.

La cuarta, referida a lo que eliminarían, decían: el sentido de los MdR pues eran "pura fachada", además prescindirían de los confidentes, de los internos no comprometidos, de las normas estúpidas, de que los internos se puntuaran entre ellos $y$, en el deporte, de la musculación y del boxeo.

Las mejoras se centraban en que todos tuvieran la misma oportunidad de estar, incluso los internos complicados o los funcionarios que lo desearan -no los "enchufados"-, más personal y medios, más formación profesional y educación y más trabajo productivo, compromiso con la formación y el trabajo, más talleres productivos, más cursos, reconocer el trabajo del funcionario, colaboración entre el equipo técnico y funcionario, más seguimiento del interno en su actitud y aptitud, quitar poder a los internos, menos paternalismo, selección más exhaustiva, más salidas programadas y mayor control de las ONGs. 
Y por último, la evolución de los MdR conformaba otro aspecto clave. La opinión de los tres sectores era que estos módulos debían reorientarse a la formación profesional, a la formación en valores, a un ambiente de estudios regulado y a un trabajo regulado y productivo. Consolidación de hábitos laborales, educativos, formativos y superación de conflictividad al respeto mutuo. No limitar los MdR a pautas de convivencia, ir hacia la reinserción y reintegración, es decir, -y en palabras de los profesionales- que haya una "necesaria evolución, pues actualmente solo importan las normas de urbanidad". Exigencia de coordinación, porque los MdR se estaban convirtiendo en "fábricas de simulación de conducta". Era necesaria la concienciación del interno sobre la problemática que le ha llevado a prisión.

\section{Discusión y conclusiones}

El objetivo de este estudio es explorar la opinión de los distintos profesionales que trabajan en el ámbito penitenciario (funcionarios de vigilancia, equipo técnico y mando directivo) sobre los MdR.

Tras el análisis de los datos numéricos, apreciamos relaciones significativas sobre la consideración de los MdR como herramienta formativa y sobre su concepto, pero no sobre la utilidad ni su viabilidad.

No nos sorprenden estos dos últimos resultados, pues la utilidad, tal y como se recoge también tras el análisis cualitativo de la información con las preguntas abiertas, no se cuestiona, ya que los MdR (ver anexo) suponen una

concepción menos punitiva de la prisión y más educativa, basada en el compromiso voluntario de los internos de cumplir las normas y pautas de conductas propias de la convivencia, no tanto en el sentido de disciplina sino de relaciones sociales con los demás internos y de hábitos personales de higiene o no consumo de drogas (Cervelló, 2016, p. 271),

de cuidado del entorno y de relación interpersonal (Casado, 2013). El "propio funcionamiento del MdR, favorece la reinserción social" (Galán, 2015, p. 325). Tampoco la viabilidad se pone en entredicho, no en vano ya están en marcha 257 módulos distribuidos en 68 centros a nivel nacional, ubicando a 19289 internos (SGIP, 2016).

En cambio, el concepto sobre los MdR es muy valorado por los funcionarios y por los equipos técnicos. El hecho de que estas figuras profesionales crean en los MdR es fundamental para su buen desarrollo y éxito. Como sugieren Lerman y Page (2016), las perspectivas de los funcionarios penitenciarios afectan su comportamiento en el lugar de trabajo, con consecuencias en las relaciones entre el personal y los presos, la aplicación de las políticas y las operaciones rutinarias de los centros penitenciarios. Galán (2015, p. 321) argumenta que

los profesionales que forman parte de un módulo de respeto, son parte indispensable del programa, ya que se encargan de supervisar el correcto funcionamiento del módulo. Además, es necesario un mayor grado de implicación por parte de este colectivo para conseguir que el módulo de respeto sea una alternativa viable para aquellos reclusos que quieren convivir bajo unas condiciones más adecuadas dentro de prisión.

Belinchón (2009) recoge expresamente, en su Manual sobre los MdR, que es imprescindible la implicación directa y permanente del Director del centro como impulsor del proyecto; así como también del Equipo Técnico, responsable de la gestión diaria.

Como herramienta formativa, son los funcionarios y el equipo técnico los que ven suficientes y muchas, respectivamente, las actividades académicas frente al mando directivo que no tiene claro si hay bastantes o pocas. Este resultado es coherente con la reivindicación de que los MdR deben reorientarse, superando -que no abandonando- el sesgo académico, a favor de una línea más profesionalizadora, diseñar talleres ocupacionales y más trabajo productivo, así como evolucionar hacia la formación en valores (Del Pozo \& Añaños, 2013; Gil, 2010). Se trataría de "diseñar actividades de ocupación exigentes, favorecedoras del cambio personal y social, tanto en actitudes como en valores" (Gil, 2016, p. 61)

La idea de que existen pocos o ningún taller de trabajo productivo en los que los internos estén dados de alta en la Seguridad Social y sean remunerados, es una consideración en la que coinciden todos. $Y$ realmente, los tres reivindican su puesta en marcha por los beneficios que implican para el interno. El carácter socioeconómico es uno de ellos, pues supondrá una remuneración económica (Viedma \& Frutos, 2012); el penitenciario es otro beneficio que (Galán, 2015, p. 155) le permitirá "dignificarse como persona, preparar su futura salida en libertad, evitar la desocupación y, ante todo, canalizar el grado de ansiedad y estrés que genera el encierro, llegando a concebir la relación laboral, como elemento esencial en su vida diaria"; y otro es la función educativa y terapéutica que se presuponen tienen estos talleres (Esteban, Alós, Jódar \& Miguélez, 2014). Según los anteriores autores los beneficios son múltiples, pues “los internos interiorizan actitudes asociadas al 
trabajo" (p.192) como la puntualidad, responsabilidad o quehacer y porque mitiga la cultura carcelaria. La función educativa del trabajo es una idea asociada a la necesidad de potenciar la formación general, profesional y ocupacional por el proceso de socialización a través de la educación y porque el trabajo en la cárcel es la primera experiencia laboral consistente de algunos presos (Alós, Martín, Miguélez \& Gibert, 2009). Parece que se denota falta de una correcta gestión a la hora de organizar estos talleres, como apunta Salamanca (2016) quien pone de manifiesto que se contabilizan 12.500 presos trabajadores, pero hay instalaciones para 20.000 , es decir, se cuenta con talleres equipados y vacíos porque no hay empresas que lleven allí su trabajo.

Las actividades deportivas que se programan en los MdR, para los tres sectores, son suficientes. Sin duda las consideran beneficiosas. Este hecho lo corroboran los estudios que hay sobre el deporte en los centros penitenciarios, cuyos resultados demuestran los beneficios para la convivencia y el autocontrol en los internos (Castillo, 2005). Aunque estos estudios no diferencian entre deportes, los funcionarios prefieren eliminar las salas de musculación y boxeo y apostar por otro tipo que no suponga la cultura al cuerpo.

Que se desarrollen cursos formativos o de inserción laboral son reconocidos como suficientes por funcionarios y equipo técnico y como escasos por los mandos directivos. Como defienden Del Pozo y Añaños (2013) la educación es un reto, un derecho y un principio deontológico. Martín (2008, p. 23) está a favor de las “intervenciones pedagógicas que conduzcan hacia la reeducación", es decir, según sus palabras, el componente pedagógico y educativo deben presidir las actuaciones de los centros penitenciarios.

¿Discrepamos de nuestros resultados cuantitativos? En absoluto. Esta es la situación que muestra la realidad andaluza vista por los distintos sectores. Pero también merece la pena discutir la visión obtenida a raíz de las categorías definidas a partir de las preguntas abiertas.

Lo mejor de los MdR es que logra todos y cada uno de los objetivos para los que fue creado y así lo reconocen los tres sectores: participación, autonomía, salubridad, respeto y entorno libre de droga. Son los ejes sobre los que se vertebran estos módulos y así se reconoce (Belinchón, 2009; Belinchón \& García, 2014; Cendón, Belinchón \& García, 2011).

Lo peor es la opinión, por parte de los funcionarios y equipos técnicos, de la simulación de las conductas de los internos. Por tanto, habría que revisar el sistema de premios y quién administra los premios que logran los internos (Valderrama,
2016). Los funcionarios cuestionan que estos puedan sancionarse entre ellos, considerando que habría que retirarles este poder y revisar su sobre-autoridad.

También piensan que hay que cambiar el sistema de acceso al módulo, porque entienden que, realmente, no se incluye a todos los perfiles de internos. Esta idea es muy importante. Si los que trabajan dentro del módulo tienen reservas sobre el tipo de acceso, habría que reflexionar, pues como apunta Galán (2015, p. 365), las personas ajenas a estos módulos, funcionarios e internos, conviven con la premisa de que el "programa aloja a chivatos, violadores y protegidos dentro de prisión" y será una variable que perturbe su desarrollo.

Lo que modificarían son las relaciones de poder entre funcionarios y equipos técnicos. Se reconoce la existencia de falta de concienciación del personal penitenciario y descoordinación de los distintos estamentos (Añaños \& Yagüe, 2013). La formación específica y la delimitación clara de funciones y responsabilidades allanan el terreno. Galán (2015) defiende que, tanto funcionarios como equipo técnico, han de encargarse de que el MdR funcione, además de evaluar el comportamiento del interno y tomar las decisiones pertinentes. Según Valderrama (2016) queda bien definido el campo de actuación de cada profesional, pues en los tres niveles que establece, el primero lo ocuparía el funcionario de vigilancia quien diariamente rellena una hoja de registro sobre la evolución del interno. El segundo, lo realiza el responsable de la actividad específica diaria; y el tercero, supone una evaluación semanal por parte del Equipo Técnico en la que participan los funcionarios de vigilancia.

Lo que mejorarían, especialmente los equipos técnicos, serían las salidas programadas y el mayor control de las ONGs. Y es que compartimos la idea de que es difícil acometer procesos de reinserción, segregando a la población a la que se pretende reinsertar y aislándola del contacto con la sociedad (Martín, 2008). Por otro lado, se hace una llamada de atención a la desprofesionalización educativa en el ámbito penitenciario (Gil, 2010), a la que podría contribuir el hecho de que muchas de las actividades dentro de las prisiones las ejecutan las ONGs. Nuestra reivindicación es, por tanto, la necesaria profesionalización de las actuaciones llevadas a cabo en prisión.

Por último, destacamos la unanimidad de los tres sectores al considerar que los MdR han de evolucionar hacia otros derroteros. Hay que superar la idea de "aprender normas de urbanidad" -que ya está conseguido- y de que funcionan de maravilla por la ausencia de conflictos, pues de fondo subyace que es una "fábrica de simulación de conducta". 
Realmente se trata de encarar un nuevo concepto de MdR que se base en alcanzar valores morales y éticos (Martín, 2008). Se trata de reorientar los módulos hacia una nueva "ética de la responsabilización" (Martín, Vila \& De Oña, 2013, p. 29). Es decir, implicaría una metodología educativa que pasara de la culpabilización a la responsabilidad y que trabajara con cada persona, para que se hiciera responsable de sus hechos y experiencias y vivir de forma "más comprometida" con la sociedad. En esta misma línea se pronuncia Caride y Gradaílle (2013, p. 44) sobre poner "un énfasis especial en los compromisos éticos de y hacia quienes están en las prisiones". Se trata de superar una moralina machacona y ejemplar, y como acertadamente refiere Savater (2012, p. 16), la ética "no va de aprenderse diez ni quince mandamientos, ni uno o dos códigos de buena conducta. La ética es la práctica de reflexionar sobre lo que vamos a hacer y los motivos por los que vamos a hacerlo". Sin duda todo el diseño de futuras acciones formativo-educativo-profesionales-ocupacionales debería considerar este nuevo fin "el porqué".

El posicionamiento frente a los MdR es favorable. Concluimos, a la luz de los resultados, que no existen asociaciones significativas entre ser funcionario de vigilancia, pertenecer al equipo técnico o formar parte de la dirección con la visión que tienen de la viabilidad y la utilidad de los MdR. Es decir, la opinión sobre la viabilidad y utilidad no se relaciona con pertenecer a un sector $u$ otro. En cambio, sí se asocia con entender el MdR como herramienta formativa en el que se celebran actividades académicas, talleres de trabajo productivo, actividades deportivas y cursos formativos, donde sí discrepan los tres sectores; y también existe asociación significativa con el concepto general, su inclusión y su creación, donde se reconoce que no hay unanimidad entre los sectores.

La mayor contribución de esta investigación es que analiza, a nivel autonómico, los MdR desde la perspectiva de los agentes que intervienen en su desarrollo: dirección, equipo técnico y funcionario de vigilancia. Pero sobre todo, una importante implicación del estudio es que recoge el llamamiento que los sectores estudiados hacen sobre los MdR y la necesidad de que estos evolucionen. Es necesaria una reformulación de los módulos con mayor tinte educativo y productivo, orientados hacia el compromiso con la sociedad y centrados en trabajar la "actitud" de los presos y de los profesionales por querer cambiar. Los MdR no pueden ser contenedores sociales de buenas formas, sino el lugar que prepare para la sociedad en un sentido ético y de corresponsabilidad social. Esto nos lleva irremediablemente a repensar tanto el sentido de los MdR como el perfil de los profesionales implicados en su funcionamiento, sensible este al necesario replanteamiento educativo y social del ámbito penitenciario.

Hemos de resaltar algunas limitaciones del estudio. A pesar de contar con el beneplácito de la Subdirección General de Relaciones Institucionales y Coordinación Territorial, la participación de los mandos directivos no ha sido todo lo intensa que hubiésemos deseado. Otra limitación posible es que esta investigación está circunscrita únicamente a la comunidad autónoma de Andalucía, y además, no se ha tenido en cuenta la idiosincrasia específica de los diferentes centros penitenciarios en las distintas provincias. En este sentido, hemos sido cautelosos en la discusión de los resultados y las conclusiones a la hora de referirnos a las restricciones comentadas. Quedaría abierta la posibilidad de ampliar el estudio recogiendo la voz de los internos, acercándonos a través de la investigación biográfico-narrativa, a la comprensión de los MdR desde todos los sectores.

El futuro de este trabajo pasa por comunicar sus resultados a la Subdirección de Instituciones Penitenciarias con el deseo de que "oiga la voz de las opiniones" de los distintos sectores, que tanto de forma cuantitativa como cualitativa nos han hecho llegar y hemos podido analizar. 


\section{Referencias bibliográficas}

Alós, R., Martín, A., Miguélez, F., \& Gibert, F. (2009). ¿Sirve el trabajo penitenciario para la reinserción?: un estudio a partir de las opiniones de los presos de las cárceles de Cataluña. Reis: Revista española de investigaciones sociológicas, 127, 11-32.

Antón, P. (1998). Actitudes del personal de prisiones hacia su trabajo y hacia los reclusos. (Tesis doctoral). Universidad de Deusto.

Añaños, F. (2017). En Prisión. Realidades e intervención socioeducativa y drogodependencias en mujeres. Madrid: Narcea.

Añaños, F., Fernández, P., \& Llopis, J. (2013). Aproximación a los contextos en prisión: una perspectiva socioeducativa. Pedagogía social: revista interuniversitaria, 22, 13-28. doi: 10.7179/PSRI

Añaños, F., \& Yagüe, C. (2013). Educación social en prisiones: planteamientos iniciales y políticas encaminadas hacia la reinserción desde la perspectiva de género. Pedagogía social: revista interuniversitaria, 22, 7-12. doi: 10.7179/PSRI

Belinchón, E. (2009). Módulos de Respeto. Manual. Madrid: Secretaría General de Instituciones Penitenciarias. Retrieved from https://goo.gl/cvs2x3

Belinchón, E., \& García, H. (2014). Módulos de respeto. Fundamentos metodológicos. Definición y objetivos. En A. De Juanas (Coord.), Educación social en los centros penitenciarios (153-176). Madrid: UNED.

Bisquerra, R. (1987). Introducción a la estadística aplicada a la investigación educativa. Un enfoque informático con los paquetes BMDP y SPSS. Barcelona: PPU.

Caride, J., \& Gradaílle, R. (2013). Educar en las cárceles: nuevos desafíos para la educación social en las instituciones penitenciarias. Revista de educación, 360, 36-47. doi: 10.4438/1988-592X-0034-8082-RE

Casado, J. (2013). Visión del sistema penitenciario español. Intervención psicoeducativa en la desadaptación social: IPSE-ds, 6, 41-54.

Castillo, J. (2005). Deporte y reeducación de conductas antisociales en prisión: principales experiencias. En J. Aquesolo (Eds.), Actas del Congreso Internacional de Historia del Deporte. Sevilla: Aquesolo Vegas. Retrieved from https:// goo.gl/EyK6sZ

Cea, A. (2004). Métodos de encuesta. Teoría y práctica, errores y mejora. Madrid: Síntesis.

Cendón, J., Belichón, E., \& García, H. (2011). Módulos de Respeto. Manual de aplicación. Madrid: Ministerio del Interior. Secretaría General Técnica. Retrieved from https://goo.gl/qj6Uwz

Cervelló, V. (2016). Derecho Penitenciario. Valencia: Tirant Lo Blanch.

Cohen, L., \& Manion, L. (1990). Métodos de investigación educativa. Madrid: La Muralla.

Constitución Española. Boletín Oficial del Estado 311, 29 de diciembre de 1978.

Cook, J., McClure, S., Koutsenok, I., \& Lord, S. (2008). The Implementation of Inmate Mentor Programs in the Correctional Treatment System as an Innovative Approach. Journal of Teaching in the Addictions, 7(2), 123-32. doi: http:// dx.doi.org/10.1080/15332700802418758

Del Pozo, F. (2013). Las políticas públicas para las prisiones: una aproximación a la acción social desde el modelo socioeducativo. Revista de humanidades, 20, 63-82. doi: http://dx.doi.org/10.5944/rdh.20.2013

Del Pozo, F., \& Añaños, F. (2013). La Educación Social Penitenciaria: ¿De dónde venimos y hacia dónde vamos? Revista complutense de educación, 24(1), 47-68.

Dirección General de Instituciones Penitenciarias (2007). Módulos de Respeto. Módulos penitenciarios para la mejora de la convivencia. Madrid: Ministerio del Interior. Secretaría General Técnica. Retrieved from https://goo.gl/ jQMqnR

Esteban, F., Alós, R., Jódar, P., \& Miguélez, F. (2014). La inserción laboral de ex reclusos. Una aproximación cualitativa. Reis: Revista española de investigaciones sociológicas, 145, 181-204. doi: http://dx.doi.org/10.5477/cis/reis.145.181

Expósito, E., \& Llopis, N. (2016). El tránsito del educador social en prisiones: entre el control y la transgresión. RES: Revista de Educación Social, 22, 69-84.

Galán, D. (2015). Los Módulos de Respeto: una alternativa al tratamiento penitenciario. (Tesis doctoral). Universidad Complutense de Madrid. Retrieved from https://goo.gl/NWYGSD

García-Legaz, M., \& Crespo, Álvaro (2014). Cuestionario calidad de vida en los centros penitenciarios. Madrid: Comisión de Calidad de Vida del Consejos Social Penitenciarios. Retrieved from https://goo.gl/J8CVNv

Gil, F. (2010). La acción pedagógica en las prisiones: posibilidades y límites. Revista española de pedagogía, 245, 49-66.

Gil, F. (2013). Derechos humanos y reeducación en las prisiones. El derecho a la educación en el modelo good lives. Revista de educación, 360, 48-68. doi: 10.4438/1988-592X-0034-8082-RE

Gil, F. (2016). Decálogo pedagógico de la educación social en prisión. RES. Revista de Educación Social, 50-69.

Guy, R. (2011). Applied sociology and prisoner reentry a primer for more successful reintegration. Journal of Applied Social Science, 5 (2), 40-52. doi: 0.1177/193672441100500204 
Kelchtermans, G. (1993). Getting the story, understanding the lives: From career stories to teachers' professional development. Teaching and Teacher Education, 9(5), 443-56. doi: https://doi.org/10.1016/0742-051X(93)90029-G

Lerman, A., \& Page, J. (2016). Does the Front Line Reflect the Party Line? The Politicization of Punishment and Prison Officers' Perspectives Towards Incarceration. The British Journal of Criminology, 56(3), 578-601. doi: https://doi. org/10.1093/bjc/azv061

Ley Orgánica 1/1979, de 26 de septiembre, General Penitenciaria. Boletín Oficial de Estado 239, 5 de octubre de 1979.

Lorenzo, M., Aroca, C., \& Alba, J. (2013). La pedagogía penitenciaria en España: luces y sombras. Revista de educación, 360, 119-39. doi: 10.4438/1988-592X-0034-8082-RE

Marlow, E., Grajeda, W., Lee, Y., Young, E., Williams, M., \& Hill, K. (2015). Peer Mentoring for Male Parolees: A CBPR Pilot Study. Progress in Community Health Partnerships: Research, Education, and Action, 9(1), 91-100. doi: https:// doi.org/10.1353/cpr.2015.0013

Martín, V. (2008). Estudio socioeducativo de los jóvenes internados en las prisiones andaluzas. Revista Española de Investigación Criminológica: REIC, 6, 1-25.

Martín, V., Vila, E., \& De Oña, J. (2013). La investigación educativa en el ámbito de las instituciones penitenciarias: panorámica, desafíos y propuestas. Revista de educación, 360, 16-35. doi: 10.4438/1988-592X-0034-8082-RE

Mcmillan, J., \& Schumacher, S. (2005). Investigación educativa. Madrid: Pearson.

Novo-Corti, I., \& Barreiro-Gen, M. (2015). Walking From Imprisonment Towards True Social Integration: Getting a Job as a Key Factor. Journal of Offender Rehabilitation, 54 (6), 445-464. doi: http://dx.doi.org/10.1080/10509674.2015.1 055036

Quispe, A. (2014). Procesamiento, análisis e interpretación de datos sociales. En A. Díaz, \& A. Luna (eds.), Metodología de la investigación educativa: Aproximaciones para comprender sus estrategias (141-158). México: Díaz de Santos.

Rodríguez, M., Olmos, S., \& Martínez, F. (2012). Propiedades métricas y estructura dimensional de la adaptación española de una escala de evaluación de competencia informacional autopercibida. Revista de Investigación Educativa, 30 (2), 347-366. doi: http://dx.doi.org/10.6018/rie.30.2.120231

Salamanca, L. (2016). Intervención educativa en prisión: memoria y deseo. RES, Revista de Educación Social, 22, 159-73.

Savater, F. (2012). Ética de urgencia. Madrid: Ariel.

Scarfó, F., Breglia, F., \& López, C. (2016). El aporte de la Pedagogía Social en la formación de los/as funcionarios/as penitenciarios. RES, Revista de Educación Social, 22, 85-98.

SGIP (2016). Informe General 2015. Madrid: Ministerio del Interior. Retrieved from https://goo.gl/ugoaYf

Suhling, S. \& Guéridon, M. (2016). Comparisons between social therapeutic institutions. Forens Psychiatr Psychol Kriminol, 10 (32). doi:10.1007/s11757-015-0355-y

Tagliacarne, G. (1968). Técnica y práctica de la investigación de mercado. Barcelona: Ariel.

Thorndike, R. (1997). Measurement and Evaluation in Psychology and Education. New York: Macmillan.

Valderrama, P. (2010). Cárcel: poder, conflicto y ciudadanía. La micropolítica de la función reeducadora: La micropolítica de la función reeducadora. Málaga: Universidad de Málaga.

Valderrama, P. (2013). La micropolítica de la función reeducadora en prisión. Revista de educación, 360, 69-90. doi: 10.4438/1988-592X-0034-8082-RE

Valderrama, P. (2016). Los módulos de respeto en las cárceles, una revisión desde la Educación Social. RES, Revista de Educación Social, 22, 29-49.

Viedma, A., \& Frutos, D. (2012). El trabajo en prisiones: Observando las desigualdades de género. En C. Del Val, \& A. Viedma (Coords.), Condenadas a la desigualdad: sistema de indicadores de discriminación penitenciaria (87-108). Barcelona: Icaria. 


\section{Anexo}

\section{Módulo de respeto, cultura, educación y deporte. Centro Penitenciario de Albolote (Granada)}

\section{Normas de convivencia en el módulo 13}

1a Debes cuidar tu aspecto utilizando el vestuario adecuado para cada ocasión, siendo obligatorio la ducha diaria y el cambio de ropa interior.

2a Queda terminantemente prohibido el consuma de toda clase de drogas.

za Sólo se permite fumar en las dependencias autorizadas (patio y celda).

$4^{a}$ La celda estará en perfecto estado de limpieza y ordenada, teniendo el vestuario debidamente colocado en su lugar habilitado.

Diariamente se pasará revista a la celda. En caso de incumplimiento de la normativa, los internos que se alojen en la misma obtendrán una valoración negativa que se reflejará en la "hoja de valoración" y se tendrá en cuenta para la evaluación semanal.

$5^{a}$ Queda prohibido cualquier almacenamiento de alimentos en las celdas con la excepción de dos piezas de fruta y dos botella de agua por interno.

Se autoriza a lavar la ropa en la celda.

6a No arrojar nada al suelo, ni escupir, utilizando las papeleras y ceniceros. Quedando terminantemente prohibido arrojar basura y objetos por la ventana de la celda.

7a No se permite el acceso a la celda de otro interno sin estar el titular de la misma presente.

8a Al toque de diana se levantará el interno, se aseará y pondrá en orden su celda. Durante los recuentos, el interno estará de forma respetuosa.

qa La asistencia y puntualidad a todo tipo de actividades, asambleas o reuniones es muy importante.

10a El interno respetará los turnos establecidos (comida, teléfono, economato, consultorios, etc.). En el comedor no hay sitio propiedad del nadie, no se puede entrar con ropa deportiva ni chanclas de goma, tampoco con camiseta de tirantes o cortadas.

11a En horario de actividades, no se puede subir a las celdas sin autorización de los funcionarios y por algún motivo serio.

12a El interno tendrá muy presente el espíritu de fomentar el objetivo del módulo, basado en el RESPETO, LA CULTURA Y EL DEPORTE.

El incumplimiento de las presentes normas de convivencia será motivo de estudio por los miembros del EQUIPO TÉCNICO, y en su caso, la expulsión.

En Albolote, a de de 20

Fdo. El interno: 


\section{CÓMO CITAR ESTE ARTÍCULO}

Fernández, F., Pérez-García, P. (2018). La opinión de los profesionales de los centros penitenciarios de Andalucía sobre los Módulos de Respeto. Pedagogía Social. Revista Interuniversitaria, 31, 169-182. DOI: 10.7179/PSRI_2018.31.13

\section{DIRECCIÓN COMPLETA DE LOS AUTORES}

Francisco Fernández Millán. C/ Fátima 22. Las Gabias 18110 (Granada. España). - Email: franciscofmillan@hotmail.com

Purificación Pérez-García. Campus Universitario de Cartuja. Facultad de Ciencias de la Educación s/n18071 Granada mpperez@ugr.es

\section{PERFIL ACADÉMICO}

Francisco Fernández Millán. Doctor en Ciencias de la Educación y Licenciado en Derecho, ambas titulaciones por la Universidad de Granada. Título de Máster Universitario en Dirección de Centros Penitenciarios por la UNED. Funcionario de Prisiones desde el año 1989, actualmente desempeña el puesto de Educador.

Purificación Pérez-García. Profesora Titular de Universidad desde 2007 en la Facultad de Ciencias de la Educación de la Universidad de Granada (España). Sus intereses de investigación están relacionados con la formación del docente, formación inicial, practicum, enseñanza universitaria y ética profesional. Ha publicado libros y artículos sobre estas líneas temáticas. 